\title{
Structural and Functional Characterization of a New Double Variant Haemoglobin (HbG-Philadelphia/Duarte $\alpha_{2}^{68 \text { Asn } \rightarrow \text { Lys }} \beta_{2}^{62 A l a ~} \rightarrow$ Pro $)$
}

\author{
Antonella Fais, ${ }^{1}$ Mariano Casu, ${ }^{2}$ Paolo Ruggerone, ${ }^{3}$ Matteo Ceccarelli, ${ }^{3}$ \\ Simona Porcu, ${ }^{1}$ Benedetta Era, ${ }^{1}$ Roberto Anedda, ${ }^{2}$ Maria Carla Sollaino, ${ }^{4}$ \\ Renzo Galanello, ${ }^{4}$ and Marcella Corda ${ }^{1}$ \\ ${ }^{1}$ Department of Sciences Applied to Biosystems, University of Cagliari, S.P. Monserrato-Sestu km 0.700, 09042 Monserrato, Italy \\ ${ }^{2}$ Department of Chemical Sciences, University of Cagliari, S.P. Monserrato-Sestu km 0.700, 09042 Monserrato, Italy \\ ${ }^{3}$ SLACS, Istituto Officina dei Materiali del CNR and Department of Physics, University of Cagliari, \\ S.P. Monserrato-Sestu km 0.700, 09042 Monserrato, Italy \\ ${ }^{4}$ Department of Biomedical and Biotecnologies Sciences, University of Cagliari, via Jenner, 09121 Cagliari, Italy
}

Correspondence should be addressed to Paolo Ruggerone, paolo.ruggerone@dsf.unica.it

Received 8 September 2010; Accepted 4 October 2010

Academic Editors: J. S. Gibson, T. Ikuta, and D. Lavelle

Copyright () 2011 Antonella Fais et al. This is an open access article distributed under the Creative Commons Attribution License, which permits unrestricted use, distribution, and reproduction in any medium, provided the original work is properly cited.

We report the first case of cosegregation of two haemoglobins (Hbs): HbG-Philadelphia [ $\alpha 68($ E17)Asn $\rightarrow$ Lys] and HbDuarte $\left[\beta 62(\mathrm{E} 6) \mathrm{Ala} \rightarrow\right.$ Pro]. The proband is a young patient heterozygous also for $\beta^{\circ}$-thalassaemia. We detected exclusively two haemoglobin variants: HbDuarte and HbG-Philadelphia/Duarte. Functional study of the new double variant HbGPhiladelphia/Duarte exhibited an increase in oxygen affinity, with a slight decrease of cooperativity and Bohr effect. This functional behaviour is attributed to $\beta 62 \mathrm{Ala} \rightarrow$ Pro instead of $\alpha 68 \mathrm{Asn} \rightarrow$ Lys substitution. Indeed, HbG-Philadelphia isolated in our laboratory from blood cells donor carrier for this variant is not affected by any functional modification, whereas purified $\mathrm{Hb}$ Duarte showed functional properties very similar to the double variant. NMR and MD simulation studies confirmed that the presence of Pro instead of Ala at the $\beta 62$ position produces displacement of the E helix and modifications of the tertiary structure. The substitution $\alpha 68($ E17)Asn $\rightarrow$ Lys does not cause significant structural and dynamical modifications of the protein. A possible structure-based rational of substitution effects is suggested.

\section{Introduction}

Structural haemoglobin $(\mathrm{Hb})$ variants typically result from a point mutation in a globin gene that produces a single amino acid substitution in the corresponding globin chain. Most of the natural and recombinant haemoglobin variants possess one or two amino acid substitutions on the same polypeptide chain [1] while Hbs with substitutions in both $\alpha$ and $\beta$ chains are only occasionally reported $[2,3]$. The availability of natural $\mathrm{Hb}$ variants offers the opportunity to directly correlate structural and functional modifications to the clinical manifestations [4-6].

Haemoglobin variants are often associated with $\alpha$ or $\beta$ thalassaemia, and, while heterozygotes for the variants can manifest limited clinical effects and may be asymptomatic, the double heterozygosity for structural variants and thalassaemia may lead to severe clinical diseases [4-8]. Therefore, a careful analysis of families with interacting haemoglobin variants and $\beta$ thalassaemia is relevant. Moreover, their identification is important for genetic counselling and for structure-function relationship studies.

Worldwide, more than 800 natural haemoglobin variants have been identified so far [1]. The modifications in their structural properties are responsible for protein instability and may lead to misfolding and precipitation, while alterations in the functional properties are generally associated with altered oxygen affinity, the extent of each phenomenon depending on the location and nature of the substituted amino acid. 
During a screening program for the identification of $\beta$ thalassemia carriers in Sardinia, we identified in a subject heterozygote for the beta zero 39 nonsense mutation a new double variant resulting from tetrameric assembly of the $\alpha^{68 \mathrm{Asn} \rightarrow \text { Lys }}$ with $\beta^{62 \mathrm{Ala} \rightarrow \text { Pro }}$ globin chains. HbDuarte $\left(\alpha_{2} \beta_{2}^{62 \mathrm{Ala} \rightarrow \text { Pro }}\right)$ and HbG-Philadelphia $\left(\alpha_{2}^{68 \mathrm{Asn} \rightarrow \text { Lys }} \beta_{2}\right)$ were previously studied [9-11] but the presence of both substitutions in the same tetramer has not been described before.

The HbG-Philadelphia/Duarte offers the rare opportunity to characterize a new naturally occurring double $\mathrm{Hb}$ variant and to provide some insights into the still open problem of the influence of point mutations on the structure-function relationships in a protein. For example, amino acid substitutions located at $\alpha 68$ (E17) position such as in HbUbe $\left(\alpha_{2}^{68 \mathrm{Asn} \rightarrow \mathrm{Asp}} \beta_{2}\right)[12,13]$ do not influence the functional properties of $\mathrm{Hb}$ molecule, while for HbGPhiladelphia two previous papers proposed opposite results $[14,15]$ : North et al. [14] reported an increased oxygen affinity not detected by Pardoll et al. [15]. HbDuarte has an oxygen affinity higher than the $\mathrm{HbA}$ and a moderate instability $[9,11]$.

Additionally, we have the chance to assign the true contribution of two specific amino acid substitutions on determining the functional and structural behaviour of the double variant compared with the single variants $\mathrm{Hbs}$ once these latter have been well characterized. In other words, we might discriminate whether the double substitutions affect additively the properties of the system, that is, the final effect is the sum of the single effects, or additional intereffects come into play subtly producing new features. Finally, it would be useful to extract some structural-dynamical criteria to foresee the perturbations introduced in the systems by multiple mutations.

Using a series of approaches, including molecular biology and protein studies, we carried out the investigation on the purified double variant haemoglobin. In addition, proton Nuclear Magnetic Resonance (NMR) spectroscopy and Molecular Dynamic (MD) simulations assessed the effects of the amino acid substitutions on the tertiary and quaternary structure, as well as on the dynamical fluctuations.

\section{Materials and Methods}

2.1. Blood Samples. Venous samples from the proband and from his parents were collected using heparin or EDTA as an anticoagulant. Cells were washed three times with an isoosmotic $\mathrm{NaCl}$ solution by centrifugation at $1000 \mathrm{~g}$, and the packed cells were lysed by adding distilled water in a $1: 1$ ratio. After incubation at $4^{\circ} \mathrm{C}, 1$ vol. of $\mathrm{CCl}_{4}$ was added, and the solution was then centrifuged for $30 \mathrm{~min}$ at $12000 \mathrm{~g}$ to remove the ghosts.

2.2. DNA Sequences of Globin Genes. DNA was extracted from EDTA peripheral blood samples with saline method. To define the mutations, DNA of $\alpha$ and $\beta$ globin genes was amplified by PCR [16] and directly sequenced using ABI PRISM DNA 3100 (Applied Biosystem).
2.3. Haemoglobin Analysis. The red cells lysate was analysed by IEF in $5 \%$ thin layer polyacrylamide gels (Pharmalyte pH range 6.7-7.7 Amersham Pharmacia Biotec AB) [17]. The abnormal haemoglobins were identified and quantified by HPLC (Variant I, Bio-Rad, Milan, Italy). Dissociated globin chains were analysed in polyacrylamide gels in the presence of 5\% acetic acid, $8 \mathrm{M}$ urea and Triton X-100 (AUTPAGE) [18-20] and by RP-HPLC (Agilent 1100 series) on a Zorbax 300SB-C18 column (Agilent Technologies). Each chromatogram was developed at room temperature with a linear gradient from $60 \%$ to $80 \%$ of the solvent A (50\% acetonitrile, $20 \%$ methanol, $30 \% \mathrm{NaCl} 155 \mathrm{mM}$ ) in solvent B (25\% acetonitrile, $40 \%$ methanol, $30 \% \mathrm{NaCl} 155 \mathrm{mM})$. The solvent program was a 90 min gradient with a flow rate of $1.4 \mathrm{~mL} / \mathrm{min}$. Absorbance was monitored at $215 \mathrm{~nm}$. HbDuarte, double variant HbG-Philadelphia/Duarte present in the proband lysate, and HbG-Philadelphia from blood cells donor carrier for this variant were purified by ionexchange chromatography (IEC) using a HiLoad Q column (Amersham Pharmacia Biotec AB). The column was first equilibrated with $20 \mathrm{mM}$ Tris- $\mathrm{HCl}$ buffer, $\mathrm{pH}$ 8.0; then, the $\mathrm{pH}$ was decreased to 7.0 with a linear gradient. Absorbance was monitored at $280 \mathrm{~nm}$. The purity of variant $\mathrm{Hbs}$ was checked by IEF and RP-HPLC. The separated fractions were analysed by means of RP-HPLC in order to highlight the presence of possible hybrids $\alpha \alpha^{68 \mathrm{Asn} \rightarrow \mathrm{Lys}} \beta_{2}^{62 \mathrm{Ala} \rightarrow \mathrm{Pro}}$.

2.4. Functional Studies. Oxygen equilibrium curves were obtained at 25 and $37^{\circ} \mathrm{C}$, in the $\mathrm{pH}$ range $7.0-8.0$, at $\mathrm{Hb}$ concentration of $\sim 80 \mu \mathrm{M}$ on a haem basis by tonometric method [21]. An average standard deviation (SD) of $\pm 3 \%$ for values of $p_{50}$ was evaluated. The $p_{50}$ is the partial pressure of the ligand at which $50 \%$ of the haems are oxygenated.

Organic phosphates were removed from $\mathrm{Hb}$ using a Sephadex G-25 column equilibrated with $100 \mathrm{mM}$ Tris/HCl buffer $\mathrm{pH} 8.0$ containing $100 \mathrm{mM} \mathrm{NaCl}$.

Functional experiments were carried out in $100 \mathrm{mM}$ Tris or BisTris/HCl buffers, containing $100 \mathrm{mM} \mathrm{NaCl}$, in the presence and in the absence of $5 \mathrm{mM}$ 2,3-diphosphoglycerate (2,3DPG). Hill graphics [22] were built by plotting each individual $\log (y / 1-y)$ versus the corresponding $\log \mathrm{pO}_{2}$, where $y / 1-y$ is the "fractional" saturation of $\mathrm{Hb}$ in oxygen, that is, the ratio between $\mathrm{Hb}$ saturated and nonsaturated in $\mathrm{O}_{2}$. The oxygen affinity in terms of $\mathrm{p}_{50}$ and cooperativity of oxygen binding as indicated by the value of the Hill coefficient $n_{50}$ values were calculated by linear regression from Hill equation for oxygen saturation levels between $40 \%$ and $60 \%$. The magnitude of the heterotrophic effects was calculated as a $\Delta \mathrm{p}_{50} \pm$ the effector.

The methaemoglobin content was calculated from optical spectrum recorded at the end of oxygen equilibrium measurements.

2.5. ${ }^{1} H$ NMR Spectroscopy Investigation. The ${ }^{1} \mathrm{H}$ NMR spectra were recorded on a Varian Unity-Inova spectrometer at a resonance frequency of $399.948 \mathrm{MHz}$. All $\mathrm{Hb}$ samples $(\sim 3 \%)$ were dissolved in $100 \mathrm{mM}$ sodium phosphate buffer $\left(10 \% \mathrm{D}_{2} \mathrm{O}\right)$ at $\mathrm{pH}$ 7.0. The experiments were performed at 


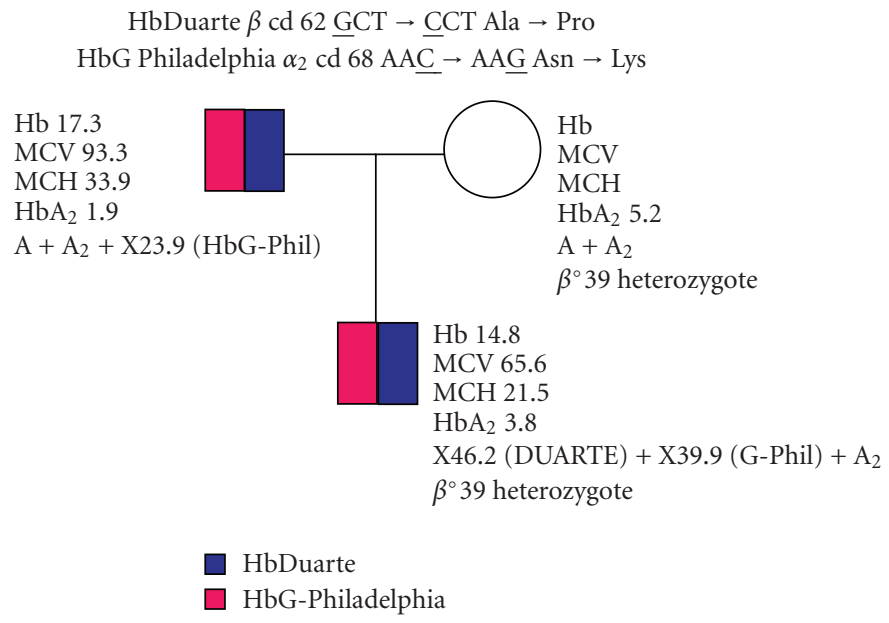

Figure 1: Family tree and haematological data.

$29.0 \pm 0.1^{\circ} \mathrm{C}$ for $\mathrm{Hb}$ in the $\mathrm{CO}$ form and in the deoxy form. All the ${ }^{1} \mathrm{H}$ NMR experiments were carried out on a $5 \mathrm{~mm}$ Wilmad high-pressure NMR tubes (OD $5 \mathrm{~mm}$ and ID $4.2 \mathrm{~mm}$ ) using $3.7 \mu \mathrm{s}$ pulse $\left(90^{\circ}\right)$, $1 \mathrm{~s}$ repetition time, and spectral width of $12 \mathrm{kHz}$ for $\mathrm{CO}$ form and $80 \mathrm{KHz}$ for deoxy form. Suppression of the intense water signal was achieved by direct saturation during the relaxation delay. The accuracies of chemical shift measurements in our samples, determined by repeating the experiments with three different samples, are $\pm 0.08 \mathrm{ppm}$ for the resonances in the $\mathrm{CO}$ form and in the range $10-25 \mathrm{ppm}$ for the resonances in the deoxy form. Chemical shifts in all spectra were referenced to DSS (2,2-dimethyl-2-silapentane-5-sulfonate) through the water signal set at $4.80 \pm 0.05 \mathrm{ppm}$.

2.6. Protein Structural Analyses. We used state-of-the-art MD simulations at an all-atom level to investigate structural rearrangements of the protein at the atomic scale. We started with the T deoxy form (PDB code: $1 \mathrm{hbhb}$; resolution $1.74 \AA$ [23]) solvated in a truncated octahedron cell with initial size of $80 \AA$, resulting in a system with 9500 water molecules ( $\sim 37000$ atoms). The simulation scheme was successfully used also in the study of myoglobin [24-26]. We refer to [11] for all details on the simulation protocol. At the end of the relaxation cycle ( $3 \mathrm{~ns}$ ), we introduced by hand the mutations. We prepared two independent systems: (i) the double variant substituting the alanine amino acid in proline at the positions E6 of the $\beta$ chains and the asparagine to lysine at position E17 of the $\alpha$ chains, (ii) the Philadelphia variant substituting only the asparagines. $\mathrm{HbA}$ and mutated systems were simulated for additional $6 \mathrm{~ns}(\mathrm{HbA}), 8 \mathrm{~ns}$ (double variant), and $1.5 \mathrm{~ns}$ (HbG-Philadelphia), saving data every $100 \mathrm{fs}$. This procedure allows us to follow relaxation of both systems and to compare them to possibly point out the structural and dynamical perturbations induced by the mutation.

\section{Results}

3.1. DNA and $\mathrm{Hb}$ Analyses. The hemolysate of proband checked by HPLC revealed the features of two main Hbs species, the first one $(\sim 46.2 \%)$ corresponding to Hb Duarte (that has a mobility like $\mathrm{HbA})$ and the second $(\sim 39.9 \%)$ to $\mathrm{Hb}$ G-Philadelphia.SLACS-Istituto Officina dei Materiali del CNR and Department of $\mathrm{HbA}_{2}$ and $\mathrm{HbF}$ accounted for $3.8 \%$ and $1.30 \%$ of the total amount, respectively (data not shown).

The family tree and the haematological data are described in Figure 1. The proband analysis of amplified DNA pointed out the presence of a nonsense mutation at codon 39 in the heterozygous state (data not shown). Direct DNA sequencing of amplified $\beta$ globin genes revealed a mutation at codon 62 GCT $\rightarrow$ CCT (Figure 2(a)) compatible with an Ala $\rightarrow$ Pro substitution, while sequencing $\alpha$ globin genes unveiled a single nucleotide substitution: $\mathrm{AAC} \rightarrow \mathrm{AAG}$ at codon 68 of $\alpha_{2}$ gene (Figure 2(b)), which results in a Asn $\rightarrow$ Lys substitution. All these experimental data, in agreement with the presence of the heterozygosity for $\beta^{0}$ thalassaemia, suggested that the hemolysate is composed by two tetramer molecules, namely, $\alpha_{2} \beta_{2}^{62 \mathrm{Ala} \rightarrow \text { Pro }}$ (HbDuarte) and $\alpha_{2}^{68 \mathrm{Asn} \rightarrow \text { Lys }} \beta_{2}^{62 \mathrm{Ala} \rightarrow \text { Pro }}$, the new double variant named HbG-Philadelphia/Duarte.

IEF procedure confirmed the presence of two haemoglobin components (Figure 3(a) lane 1), one of which had an electrophoretic mobility similar to that of HbA (Figure 3(a) lane 2), the other migrating more slowly. RP-HPLC analysis, as shown in Figure 3(b), exhibited an arrangement of the hemolysate in three globin chains, two $\alpha$ like and one $\beta$ like [27].

To further verify this hypothesis the haemoglobin components were purified by IEC and analysed by IEF (Figure 3(a) lanes 3 and 4 ). The $\mathrm{Hb}$ component that moved like HbA (Figure 3(a) lane 3) was identified as HbDuarte [9], the other component as the double variant $\left(\alpha_{2}^{68 \mathrm{Asn} \rightarrow \text { Lys }} \beta_{2}^{62 \mathrm{Ala} \rightarrow \text { Pro }}\right) \quad($ Figure 3(a) lane 4$)$. Globin chains analysis by RP-HPLC of the two haemoglobins purified supported the electrophoretic results (Figures 3(c) and 3(d)) confirming that the two haemoglobin components of the hemolysate were purified and identified as $\alpha_{2} \beta_{2}^{62 \mathrm{Ala} \rightarrow \text { Pro }}$ (HbDuarte) (Figure 3(c)) and as a double variant $\mathrm{Hb}$, consisting of $\alpha_{2}^{68 \mathrm{Asn} \rightarrow \text { Lys }} \beta_{2}^{62 \mathrm{Ala} \rightarrow \text { Pro }}$ (Figure $3(\mathrm{~d})$ ). From the 


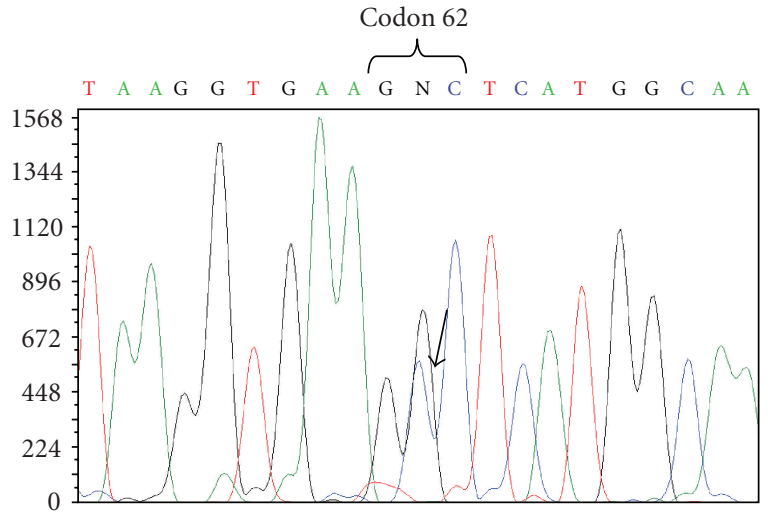

(a)

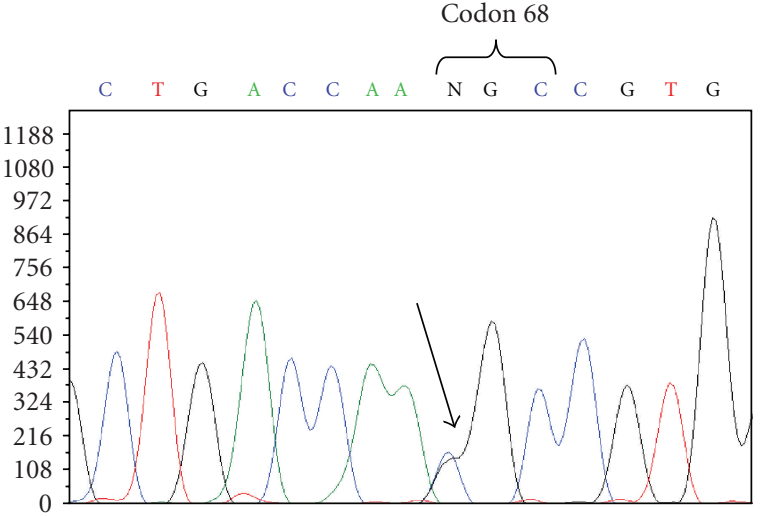

(b)

FIGURE 2: Sequence analysis (ABI PRISM 3100-Applied Biosystem) of globin genes. (a) $\beta$-globin gene coding strand of the proband showing that he was heterozygous for Hb Duarte $\underline{G C T} \rightarrow \underline{C C T}(\beta 62 \mathrm{Ala} \rightarrow$ Pro). (b) $\alpha$-globin gene coding strand of the proband showing that he was heterozygous for $\mathrm{Hb}$ G-Philadelphia $\mathrm{AA} \underline{\mathrm{C}} \rightarrow \mathrm{AAG}(\alpha 68 \mathrm{Asn} \rightarrow$ Lys).

analysis of the purified haemoglobins the presence of any hybrid species $\left(\alpha \alpha_{2}^{68 \mathrm{Asn} \rightarrow \text { Lys }} \beta_{2}^{62 \mathrm{Ala} \rightarrow \text { Pro }}\right.$ was ruled out, as it can be seen in Figures 3(a) and 3(b).

The presence of haemoglobin with both $\alpha$ and $\beta$ mutations did not produce apparently any particular clinical effect, being the patient asymptomatic. The proband presented thalassaemia-like hematological features, reduced mean corpuscular volume (MCV $65.6 \mathrm{fl}$ ), mean corpuscular haemoglobin ( $\mathrm{MCH} 21.5 \mathrm{pg}$ ), increased $\mathrm{HbA}_{2}(3.8 \%)$, reticulocytes $2.6(\times 1000)$, but haemoglobin concentration $(14,8 \mathrm{~g} / \mathrm{dl})$ was higher as compared to beta thalassemia carriers.

3.2. Functional Studies. The oxygen affinity in terms of $\log \mathrm{p}_{50}$ and cooperativity $\left(n_{50}\right)$ of purified HbG-Philadelphia/Duarte are reported in Table 1 in comparison with $\mathrm{HbA}$. The ratio of $\mathrm{p}_{50}(\mathrm{HbA}) / \mathrm{p}_{50}$ (HbG-Philadelphia/Duarte) ranged from 2.63 to 1.77 depending on $\mathrm{pH}$ and organic phosphate, this being indicative of the higher $\mathrm{O}_{2}$ affinity of the double variant $\mathrm{Hb}$. The cooperativity of oxygen binding value and the alkaline Bohr effect $\left(\Delta \log \mathrm{p}_{50} / \Delta \mathrm{pH}\right)$ measured in absence and in the presence of 2,3-DPG slightly diminished with respect to that of native human HbA.

Figure 4 reveals a shift of the Hill plot of HbGPhiladelphia/, and HbDuarte purified toward smaller values with respect to HbG-Philadephia and HbA. The data indicate that purified HbG-Philadelphia/Duarte and HbDuarte are characterized by undistinguishable $\mathrm{O}_{2}$ binding properties but different from those of purified HbG-Philadelphia and $\mathrm{HbA}$.

The amount of met- $\mathrm{Hb}$ contained in the samples, after oxygen binding experiments, ranged from $2 \%$ to $7 \%$ of total $\mathrm{Hb}$.

3.3. NMR Studies. ${ }^{1} \mathrm{H}$ NMR spectra of purified $\mathrm{Hb}$ GPhiladelphia $\left(\alpha_{2}^{68 \mathrm{Asn} \rightarrow \text { Lys }} \beta_{2}\right)$ and $\mathrm{Hb}$ G-Philadelphia/Duarte $\left(\alpha_{2}^{68 \mathrm{Asn} \rightarrow \text { Lys }} \beta_{2}^{62 \mathrm{Ala} \rightarrow \text { Pro }}\right)$ in the deoxy $(\mathrm{T})$ and CO $(\mathrm{R})$ form were compared to those of $\mathrm{HbA}$ to define the effects of amino acid substitutions on the heme environment and on subunit interface interactions.

The substitution $\alpha 68$ Asn $\rightarrow$ Lys does not modify the NMR spectra of both HbG-Philadelphia and HbGPhiladelphia/Duarte in the CO form (R state), as demonstrated in Figure 5. Instead, for the deoxy form the same amino acid substitution is responsible for remarkable modifications in the spectra, as discussed in the following.

The ${ }^{1} \mathrm{H}-\mathrm{NMR}$ spectra resonances of $\mathrm{HbA}, \mathrm{HbG}-$ Philadelphia/Duarte and HbG-Philadelphia in the deoxy form from 10 to $25 \mathrm{ppm}$ together with the recently published [11] and unveiled ${ }^{1} \mathrm{H}-\mathrm{NMR}$ spectrum of HbDuarte $\left(\alpha_{2} \beta_{2}^{62 \mathrm{Ala} \rightarrow \text { Pro }}\right)$ are reported in Figure 6 to better identify changes and similarities. The resonances in the ranges 14.5$25 \mathrm{ppm}$ and $11.5-13.5 \mathrm{ppm}$ originated from protons subjected to significant hyperfine interactions with the unpaired electrons of the $\mathrm{Fe}$ atom, that is, from protons belonging to the heme groups and/or to residues located in the heme pocket.

As shown in Figure 6, some changes were observed in the HbG-Philadelphia spectrum in the range 11.5-13.5 ppm compared to HbA: in particular, the signal at $\sim 12.2 \mathrm{ppm}$ in $\mathrm{HbA}$ is shifted to $\sim 12.6 \mathrm{ppm}$ in HbG-Philadelphia. The hyperfine shifted resonances at $\sim 12.2 \mathrm{ppm}, 14.6 \mathrm{ppm}, 21.8$, and $22.8 \mathrm{ppm}$ of the HbG-Philadelphia/Duarte spectrum exhibited modifications of the chemical shift with respect to $\mathrm{HbA}$. The last two resonances observed also in the ${ }^{1} \mathrm{H}$ NMR spectrum of the HbDuarte [11] were assigned to protons in the $\beta$ chains of deoxy HbA [28].

Clearly, most of the changes observed in the spectrum of the double variant can be ascribed to the substitution $\beta 62 \mathrm{Ala} \rightarrow$ Pro. This substitution causes an adjustment in the configuration of the amino acid residues (and/or of the heme groups) in the $\beta$ chains, which sense the hyperfine interactions with the heme iron [11]. In the range 16$21 \mathrm{ppm}$, no changes were observed, the resonances at $18.7 \mathrm{ppm}$ and at $\sim 17.1 \mathrm{ppm}$ being associated with protons in 

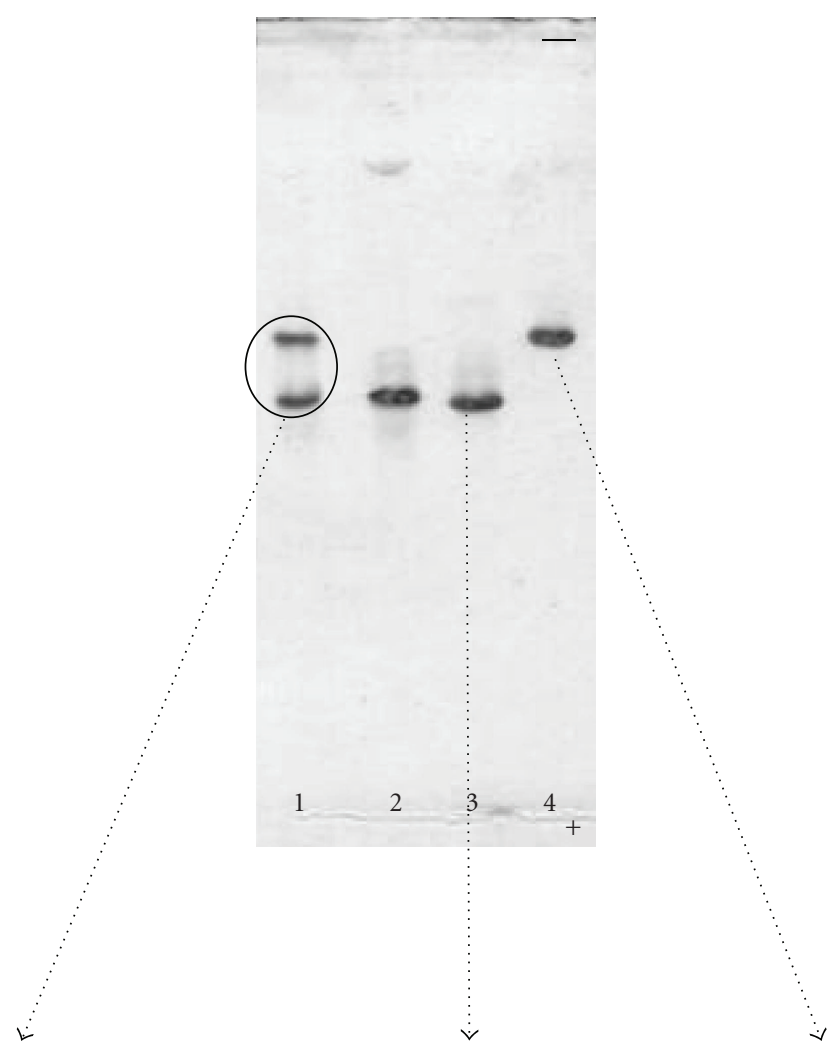

(a)

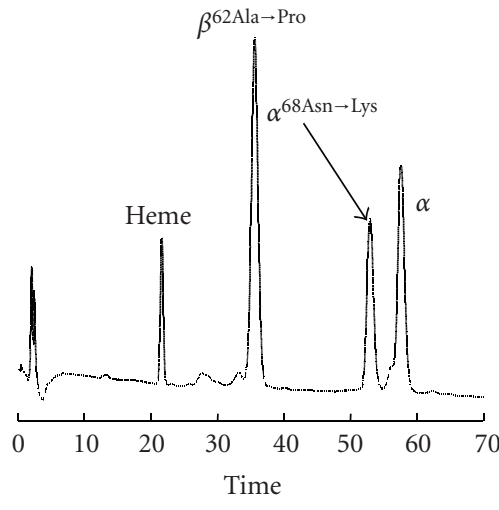

(b)

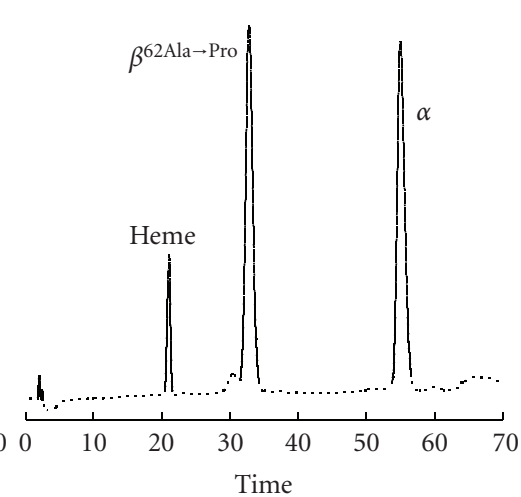

(c)

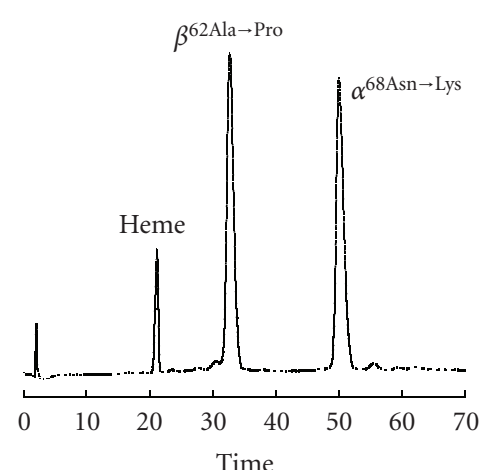

(d)

FIgURE 3: Identification of variant hemoglobins. (a) IEF of hemolysate variant (lane 1), HbA (lane 2), purified Hb Duarte (lane 3), and purified Hb G-Philadelphia/Duarte (lane 4); RP-HPLC of the globin chains of (b) hemolysate variant; (c) purified Hb Duarte; (d) purified Hb G-Philadelphia/Duarte.

the $\beta$ chains and in the $\alpha$-chains of deoxy HbA, respectively [28]. Only the shift of the signal at $\sim 12.2 \mathrm{ppm}$ in HbGPhiladelphia/Duarte was similar to that observed in HbGPhiladelphia, suggesting that structural changes in the $\alpha$ chains originate this perturbation.

Several exchangeable proton features in the range 10$15 \mathrm{ppm}$ were recognizable in the ${ }^{1} \mathrm{H}$ NMR spectrum of deoxy HbA. The resonances at $14.1 \mathrm{ppm}$ and $11.2 \mathrm{ppm}$ were identified as due to the intersubunit $\mathrm{H}$-bond between $\alpha 42$ Tyr and $\beta 99$ Asp and between $\alpha 94$ Asp and $\beta 37 \operatorname{Trp}$ in the $\alpha_{1} \beta c_{2}$ interface. The analysis of these proton resonances gave indication of the quaternary conformational modifications in the $\mathrm{T}$ state, as a consequence of residues substitutions [29-33]. In the deoxy state of $\mathrm{HbA}$ the H-bond between $\alpha 103 \mathrm{His} \rightarrow \beta 131 \mathrm{Gln}$ at the interfaces between $\alpha_{1} \beta_{1}$ subunits generated the resonance at $13.2 \mathrm{ppm}$. All these resonances in the HbG-Philadelphia spectrum coincided with those observed in the HbA spectrum, indicating that an eventual link between $\alpha_{2}^{68 \mathrm{Asn} \rightarrow \text { Lys }} \beta_{2}$ substitution and perturbations at the level of the $\alpha_{1} \beta_{1}$ and $\alpha_{1} \beta_{2}$ subunits interfaces can 
TABLE 1: Oxygen binding properties of purified HbG-Philadelphia/Duarte.

\begin{tabular}{|c|c|c|c|c|}
\hline & $\mathrm{pH}$ & $\log \mathrm{p}_{50}$ & $n_{50}$ & $\mathrm{p}_{50} \mathrm{HbA} / \mathrm{p}_{50} \mathrm{HbG}$ Philadelphia/Duarte \\
\hline \multirow{3}{*}{$-5 \mathrm{mM} 2,3-\mathrm{DPG}$} & 6.47 & $0.81(1.13)$ & $2.56(2.71)$ & 2.09 \\
\hline & 7.05 & $0.67(0.94)$ & $2.54(2.67)$ & 1.86 \\
\hline & 7.4 & $0.41(0.66)$ & $2.64(3.0)$ & 1.77 \\
\hline Alkaline Bohr effect & & $-0.43(-0.50)$ & & \\
\hline \multirow{3}{*}{ +5 mM 2,3-DPG } & 6.54 & $1.09(1.47)$ & $2.40(2.74)$ & 1.40 \\
\hline & 7.18 & $0.88(1.30)$ & $2.29(2.78)$ & 2.63 \\
\hline & 7.44 & $0.67(1.0)$ & $2.45(2.96)$ & 2.14 \\
\hline Alkaline Bohr effect & & $-0.46(-0.52)$ & & \\
\hline
\end{tabular}

Corresponding values for $\mathrm{HbA}$ are given in parentheses.

Data were obtained with $0.1 \mathrm{M}$ Bis-Tris/Tris buffer $+0.1 \mathrm{M} \mathrm{NaCl}$ at $25^{\circ} \mathrm{C} . \mathrm{O}_{2}$ pressure is expressed in Torr units.

An average S.D. of $5 \%$ for values of $\log p_{50}$ was calculed.

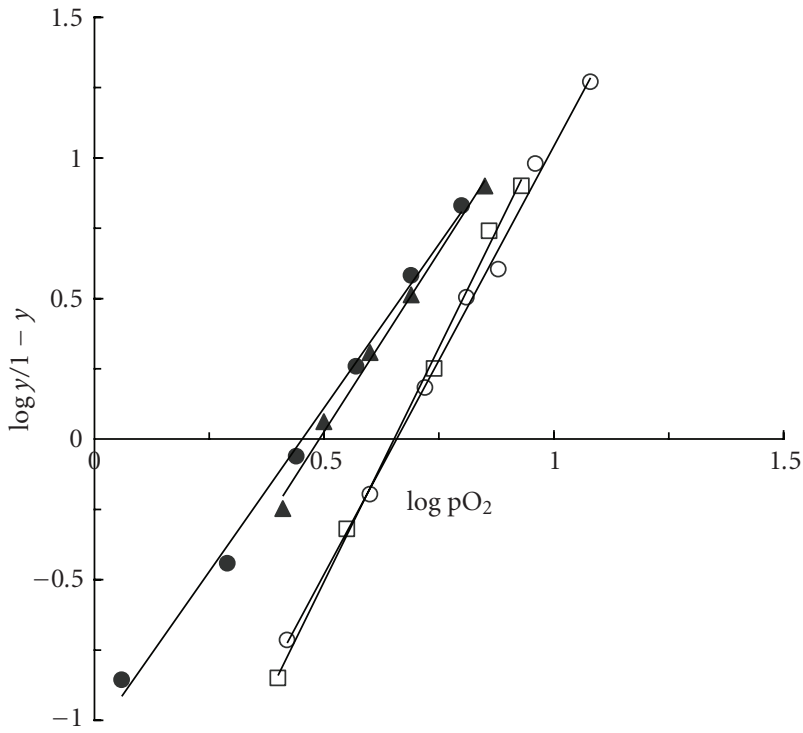

Figure 4: Hill plots of purified Hbs. $(\bullet) \mathrm{Hb}$ G-Philadelphia/Duarte, $(\boldsymbol{\Delta})$ Hb Duarte, $(\square)$ Hb G-Philadelphia and (O) HbA. Experimental conditions: $0.1 \mathrm{M}$ BisTris/Tris $0.1+0.1 \mathrm{M} \mathrm{NaCl}$ buffer at $\mathrm{pH} 7.4$, $25^{\circ} \mathrm{C} . \mathrm{O}_{2}$ pressure is expressed in Torr units.

be certainly ruled out. The resonances at $14.1 \mathrm{ppm}$ and 11.2 ppm in HbG-Philadelphia/Duarte were slightly shifted toward low fields, as already observed in the HbDuarte [11]. In agreement with what previously discussed for HbDuarte, this observation can be attributed to minor adjustments of the $\alpha_{1} \beta_{2}$ interface [11] influenced by the $\beta 62$ Ala $\rightarrow$ Pro mutation.

3.4. Structural Analyses. The substitution $\beta 62 \mathrm{Ala} \rightarrow$ Pro in HbDuarte induced structural modifications as already reported in a previous paper [11], whereas the substitution $\alpha 68$ Asn $\rightarrow$ Lys in HbG-Philadelphia does not seem to have a significant influence on the structure. In the wild type (WT), the residue $\alpha 68$ Asn forms a hydrogen bond with $\alpha 79$ Ala at position EF8, in particular with the carbonyl backbone group. The latter does not form an intrahelical hydrogen bond in the X-ray structure, Ala79 being a defect point of

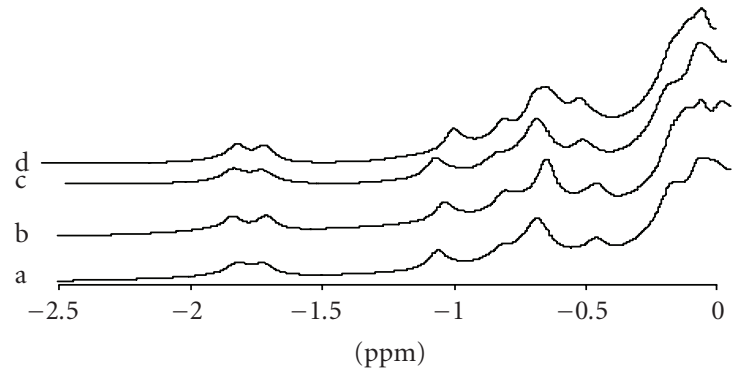

FIGURE 5: Hyperfine shifted and exchangeable proton resonances of Hbs purified in the CO form: (a) HbA, (b) Hb Duarte, (c) Hb GPhiladelphia, and (d) Hb G-Philadelphia/Durate.

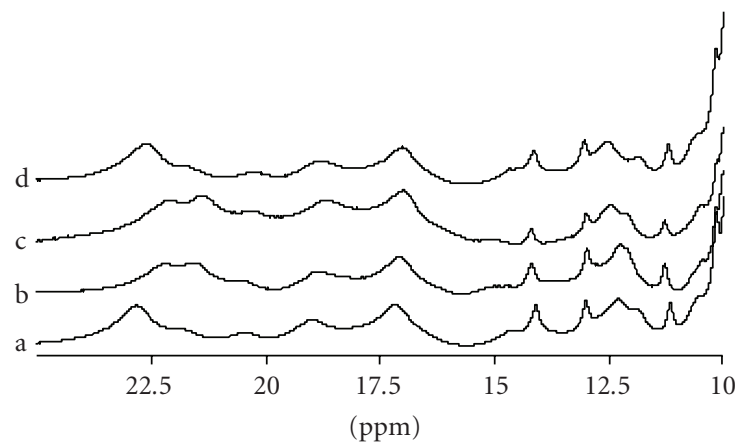

FIGURE 6: Hyperfine shifted and exchangeable proton resonances of Hbs purified in the deoxy form. (a) HbA, (b) Hb Duarte, (c) Hb G-Philadelphia/Duarte, and (d) Hb G-Philadelphia.

the helix F. Accordingly, this hydrogen bond is not constant all over our simulations, because it is detectable for only $50 \%$ of the simulation, and the interaction is not critical for both helix E and F. After substitution, being Lys a charged residue and with a longer tail, two possible hydrogen bonds can be formed either with Ala79 (30\%) or with Asp64 (70\%), at position E13 on the helix E. The lateral chain of Asp64 in WT points toward the solvent, and no crucial interaction with other amino acids is established.

As we can see from Figure 7, where we superimpose a WT structure (after $6 \mathrm{~ns}$ ) with the HbG-Philadelphia/Duarte 


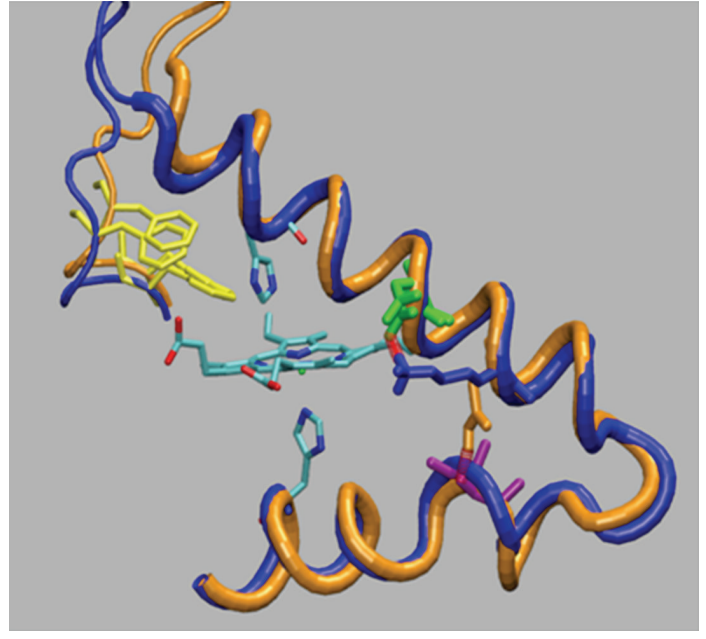

FIGURE 7: Pictorial view of the alpha globin chains superimposed after 6 and 8 ns of MD simulations. (Blue tube) double variant and (orange tube) HbA. In yellow the amino acids of the CD corner pointing the distal histidine. In green the Glu64 at position E13, in violet Ala79 at position EF8, and in orange and blue the residue at position E17, respectively, Lys and Asn, for the two systems.

structure (after $8 \mathrm{~ns}$ ), there are minimal changes in the region of $\alpha 68$. In particular, the distal region, which in the HbDuarte showed important deviations, is unaffected by the G-Philadelphia mutation (yellow amino acids in Figure 7).

Inspection of the deviations of our mutated structure from X-ray, when compared with the WT simulation, does not show any relevant deviations on the alpha subunits and on the whole tetramer (see Figure 8). The results obtained with short (1.5 ns) simulations of HbG-Philadelphia agree with the above analysis. The substitution Asn $\rightarrow$ Lys at the position E17 does not create any local rearrangements near the heme group nor at the $\alpha \beta$ interfaces.

\section{Discussion}

A structural analysis of the two variants in the proband was carried out at both protein and DNA levels, demonstrating the presence of a new haemoglobin double variant never described before, which we named HbGPhiladelphia/Duarte or $\alpha_{2}^{68 \text { Asn } \rightarrow \text { Lys }} \beta_{2}^{62 \text { Ala } \rightarrow \text { Pro }}$. The study of the proband's family pointed out that the mother was a carrier of $\beta^{0}$-thalassemia and the father of HbDuarte and HbG-Philadelphia. Only few natural haemoglobin variants carrying two substitutions in the same globin chain have been reported, and the probability to find a double mutation in the two different polypeptide chains is extremely low $[2,3]$.

The simultaneous occurrence of such a double variant in $\alpha$ and $\beta$ chains offers the excellent opportunity to investigate to which extent the effects of the double mutations sum up, once the perturbations associated with each single variant are well characterized. This could also be intended as an attempt to search structural boundaries that fix the range of influence of given mutations on tertiary and

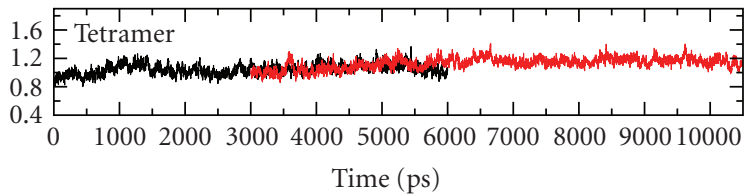

(a)

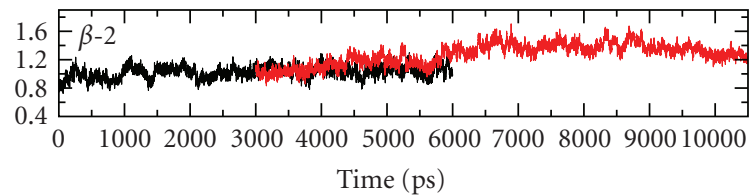

(b)

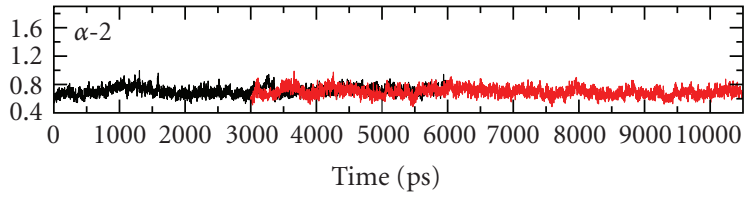

(c)

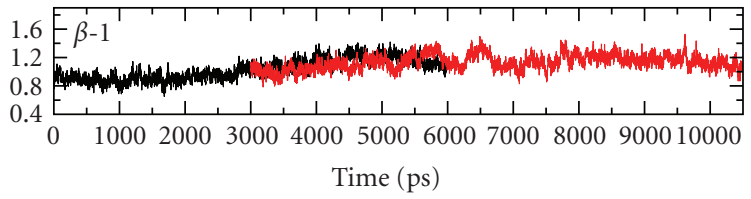

(d)

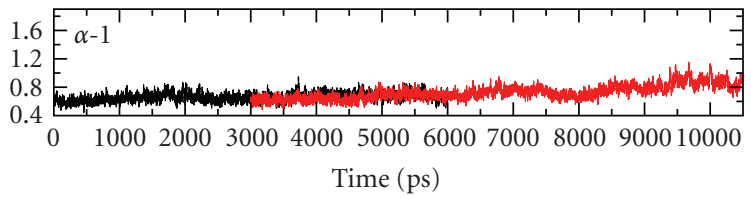

(e)

Figure 8: Xrms of (black curves) Deoxy HbA and (red curves) HbG-Philadelphia/Duarte. (a) Tetramer, (b)-(e) $\beta 2, \alpha 2, \beta 1$, and $\alpha 1$ chains, respectively.

quaternary structures. Such a study should help to map regions central to specific functionalities. Note that both substitutions considered in our study are localized in the $\mathrm{E}$ trait, which sandwiches with the F helix the heme prosthetic group. Hence, displacements in this region could weaken the $\mathrm{H}$-bonds connecting the $\mathrm{E}$ to the $\mathrm{A}, \mathrm{F}$, and $\mathrm{H}$ helices destabilizing the tertiary folding of globin chains. This might lead to a rearrangement of the heme pocket near helix $\mathrm{E}$ where oxygen binds, determining variations of functional properties. In particular, the $\alpha 68(\mathrm{E} 17)$ Asn is involved in two intrachain contacts with $\alpha 79$ (EF8)Ala and $\alpha 80(\mathrm{~F} 1)$ Leu. These contacts serve to anchor the EF clamshell holding the heme. Additionally, the E helix is connected to A helix via a linkage between $\alpha 67(\mathrm{E} 16) \mathrm{Thr}$ and $\alpha 14(\mathrm{~A} 12) \mathrm{Trp}$, and the $\mathrm{F}$ helix to $\mathrm{H}$ helix via a linkage between the backbone carbonyl group of $\alpha 93$ (FG5)Val and $\alpha 140$ (HC2)Tyr. The residue $\beta 62(\mathrm{E} 6)$, instead, is close to the distal heme-linked histidine (E7), and it is involved in maintaining the $\mathrm{E}$ helix. 
An important question is how and to which extent these two substitutions contribute to influence the structural and/or functional properties in the $\mathrm{Hb}$ tetramer.

The whole corpus of our results indicates clearly that the structural modifications responsible for the functional behaviour of the new double variant $\mathrm{Hb}$ must be ascribed exclusively to the $\beta 62 \mathrm{Ala} \rightarrow$ Pro ruling out a possible involvement of the $\alpha 68$ Asn $\rightarrow$ Lys mutation. The oxygen affinity of HbG-Philadelphia/Duarte increases; its cooperativity and the Bohr effect slightly decrease. These functional abnormalities are attributed to the single $\beta$ mutation, the same characterizing the HbDuarte, since the second substitution in $\alpha$ chain appears unable to determine functional alteration. In fact, in HbG-Philadelphia the substitution of the polar uncharged Asn residue in $\alpha 68$ with the positively charged and longer Lys does not alter the functional properties with respect to those of HbA (Figure 4). In the $\mathrm{Hb}$ Duarte the substitution of the alanine with a proline residue produces an increase in the oxygen affinity similar to the one observed in HbG-Philadelphia/Duarte [11]. These functional results are supported by extensive structural investigations via NMR and computer simulations. According to our NMR data, the $\mathrm{T}$ state and the $\mathrm{R}$ state intersubunit contacts are unaffected by the $\alpha 68$ Asn $\rightarrow$ Lys mutation in the HbG-Philadelphia/Duarte and HbG-Philadelphia. This suggests that the features of the quaternary transition $R \leftrightarrow$ $T$ are preserved upon mutation as confirmed by the almost unaltered cooperativity, DPG and Bohr effect detected in functional experiments. On the contrary, ${ }^{1} \mathrm{H}$ NMR data on the HbG-Philadelphia/Duarte shows differences in the tertiary structure around the heme pockets. In fact, the chemical shift at $\sim 12.2 \mathrm{ppm}$ attributed to the $\alpha$ chains changes upon 68 Asn $\rightarrow$ Lys mutation similarly to what observed in the HbG-Philadelphia. The other shifts observed at $\sim 14.6 \mathrm{ppm}$, $\sim 22.8$, and $\sim 21.8 \mathrm{ppm}$ in Hb G-Philadelphia/Duarte are at the same position than those observed for HbDuarte. The abnormal functional properties of HbG-Philadelphia/Duarte can be assigned to the $\beta 62 \mathrm{Ala} \rightarrow$ Pro rather than to the other substitution $\alpha 68$ Asn $\rightarrow$ Lys present in HbGPhiladelphia, which has been well demonstrated to be functionally latent (see Figure 4). In this latter haemoglobin, as in HbUbe $2[12,34]$, the site of amino acid substitution is considerably far from the distal histidine of the $\alpha$ chain (His $\alpha 58$ ), which is directly related to the function of heme iron.

A further support to the NMR analysis comes from the MD simulations that encompass microscopic features barely seen in experiments. According to MD results on HbGPhiladelphia/Duarte and HbG-Philadelphia, the pattern of intrachain contacts in the $\alpha$ chain is conserved and remains stable when Asn is substituted by Lys (Figure 7).

In tune with the search of structural thresholds identifying the effects of mutation on specific functions, the literature offers some hints: amino acid substitutions in the E trait of $\alpha$ chain do not affect significantly the functional properties [1]. Another mutant at the same position of HbGPhiladelphia, HbUbe $2\left(\alpha_{2}^{68 \mathrm{Asn} \rightarrow \mathrm{Asp}} \beta_{2}\right)$ [12, 34], presents unaltered affinity despite the change of charge carried by residue at $\mathrm{E} 17 \alpha$. It seems very difficult to modify functional- ities by acting on the trait $\mathrm{E}$ of the intrahelix contacts, which maintain tightly the $\alpha$ chain tertiary structure.

The situation is profoundly different for the $\beta$ chains: $\beta$ substitutions in $\mathrm{E}$ trait only occasionally do not affect a functional behaviour [1]. In addition, since proline can only be tolerated as one of the first three residues of a regular helix, its introduction in the middle of a helical segment usually leads to a destabilization of the subunit. In most cases, this results in a modification of functional properties and/or stability of the tetramer as indicated by several investigations on natural $\mathrm{Hb}$ variants $[1,35]$. This is the situation characterizing the double variant investigated here. The proline at the $\beta 62$ (E6) position, close to the distal haem-linked histidine (E7), produces a destabilization of the E helix toward the E1 residue that extends downward to the CD corner. The outcome is an alteration of the haem cavity structure remarkably mirrored in the functional properties of the protein molecule.

\section{Conclusions}

In conclusion, the results on the double variants and those available for the single mutations permit to point out some aspects: (1) both mutations take place in the E trait; the $\beta$ one influences structural and functional properties that in the $\alpha$ chain are practically silent; (2) the $\alpha$ and $\beta$ substitutions in HbG-Philadelphia-Duarte act independently; (3) along the E trait the functional perturbation is strictly related to the location of the substitution: the 68Asn $\rightarrow$ Asp mutation in $\alpha$ chain do not give apparent changes; the 62Ala $\rightarrow$ Pro in the $\beta$ chain give remarkable effects.

Our data and the information available to our knowledge in the literature suggest that the relative position on the globin chains (at least in the E trait), rather than the kind of amino acid substitution, plays a more important role in determining structural and functional characteristics of the haemoglobin molecule. However, the identification of a set of residues whose substitution, single or combined, causes functional and structural changes in different degree, could be useful for engineering an $\mathrm{Hb}$ variant suitable as an artificial oxygen carrying fluid.

\section{Abbreviations}

Hb: Haemoglobin

HbA: Human adult haemoglobin

NMR: Nuclear Magnetic Resonance

MD: Molecular dynamics

WT: Wild type

Xrms: Instantaneous mean-square deviation

of $\mathrm{C}_{\alpha}$ carbons from their X-ray

structure

IEF: Isoelectric focusing.

\section{Acknowledgments}

Computational resources have been granted by CINECA (INFM grant) and CASPUR (SLACS collaboration). This paper makes use of results produced by the Cybersar Project 
managed by the Consorzio COSMOLAB, a project cofunded by the Italian Ministry of University and Research (MUR) within the Programma Operativo Nazionale 20002006 "Ricerca Scientifica, Sviluppo Tecnologico, Alta Formazione" per le Regioni Italiane dell'Obiettivo 1 (Campania, Calabria, Puglia, Basilicata, Sicilia, Sardegna)-Asse II, Misura II.2 "Società dell'Informazione," Azione a "Sistemi di calcolo e simulazione ad alte prestazioni." More information is available at http://www.cybersar.it/.

\section{References}

[1] B. Giardine, S. van Baal, P. Kaimakis et al., "HbVar database of human hemoglobin variants and thalassemia mutations: 2007 update," Human Mutation, vol. 28, no. 2, p. 206, 2007.

[2] S. Fucharoen, Y. Changtrakun, T. Ratanasiri, G. Fucharoen, and K. Sanchaisuriya, "Complex interaction of Hb Hekinan $[\alpha 27(\mathrm{~B} 8) \mathrm{Glu} \rightarrow \mathrm{Asp}]$ and $\mathrm{Hb} \mathrm{E}[\beta 26(\mathrm{~B} 8) \mathrm{Glu} \rightarrow$ Lys] with a deletional $\alpha$-thalassemia 1 in a Thai family," European Journal of Haematology, vol. 70, no. 5, pp. 304-309, 2003.

[3] K. Sanchaisuriya, S. Chunpanich, S. Fucharoen, G. Fucharoen, P. Sanchaisuriya, and Y. Changtrakun, "Association of Hb QThailand with homozygous $\mathrm{Hb} \mathrm{E}$ and heterozygous $\mathrm{Hb}$ Constant Spring in pregnancy," European Journal of Haematology, vol. 74, no. 3, pp. 221-227, 2005.

[4] H. Wajcman and J. Riou, "Globin chain analysis: an important tool in phenotype study of hemoglobin disorders," Clinical Biochemistry, vol. 42, no. 18, pp. 1802-1806, 2009.

[5] B. J. Wild and B. J. Bain, "Detection and quantitation of normal and variant haemoglobins: an analytical review," Annals of Clinical Biochemistry, vol. 41, no. 5, pp. 355-369, 2004.

[6] H. Wajcman, J. Traeger-Synodinos, I. Papassotiriou et al., "Unstable and thalassemic $\alpha$ chain hemoglobin variants: a cause of $\mathrm{Hb} \mathrm{H}$ disease and thalassemia intermedia," Hemoglobin, vol. 32, no. 4, pp. 327-349, 2008.

[7] K. Adachi, S. Surrey, H. Tamary et al., "Hb Shelby $[\beta 131(\mathrm{H} 9) \mathrm{Gln} \rightarrow$ Lys] in association with $\mathrm{Hb} S[\beta 6(\mathrm{~A} 3) \mathrm{Glu} \rightarrow$ Val]: characterization, stability, and effects on $\mathrm{Hb} S$ polymerization," Hemoglobin, vol. 17, no. 4, pp. 329-343, 1993.

[8] T. H. J. Huisman, "Combinations of $\beta$ chain abnormal hemoglobins with each other or with $\beta$-thalassemia determinants with known mutations: influence on phenotype," Clinical Chemistry, vol. 43, no. 10, pp. 1850-1856, 1997.

[9] E. Beutler, A. Lang, and H. Lehmann, "Hemoglobin Duarte: $\left(\alpha_{2} \beta_{2}\right.$ 62(E6)Ala $\rightarrow$ Pro): a new unstable hemoglobin with increased oxygen affinity," Blood, vol. 43, no. 4, pp. 527-535, 1974.

[10] C. Baglioni and V. M. Ingram, "Abnormal human haemoglobins V. Chemical investigation of haemoglobins A, G, C, X from one individual," Biochimica et Biophysica Acta, vol. 48, no. 2, pp. 253-265, 1961.

[11] M. Ceccarelli, P. Ruggerone, R. Anedda et al., "Structurefunction relationship in a variant hemoglobin: a combined computational-experimental approach," Biophysical Journal, vol. 91, no. 9, pp. 3529-3541, 2006.

[12] M. Takaoki, I. Iwao, Y. Kiyomi, O. Yuzo, and S. Susumu, "Amino acid substitution of hemoglobin ube $2\left(\alpha_{2}^{68 \text { asp }} \beta_{2}\right)$ : an example of successful application of partial hydrolysis of peptide with 5\% acetic acid," Clinica Chimica Acta, vol. 16, no. 3, pp. 347-352, 1967.
[13] I. Iuchi, S. Shimasaki, K. Hidaka, S. Ueda, and S. Shibata, "Hb Ube-2 $(\alpha 68$ [E-17]Asn $\rightarrow$ Asp): the second instance in Japan," Hemoglobin, vol. 5, no. 6, pp. 599-603, 1981.

[14] M. L. North, M. C. Garel, J. Thillet, A. Gardea, J. M. Levy, and J. Rosa, "Clinica, structural and functional studies of $\mathrm{Hb}$ G-Philadelphia detected in a Maroccan newborn," Nouvelle Revue Francaise d'Hematologie, vol. 18, no. 3, pp. 601-610, 1977.

[15] J. A. Pardoll, S. Charache, B. L. Hjelle et al., "Homozygous $\alpha$ thalassemia/HbG-philadelphia," Hemoglobin, vol. 6, pp. 503$515,1982$.

[16] R. K. Saiki, D. H. Gelfand, S. Stoffel et al., "Primer-directed enzymatic amplification of DNA with a thermostable DNA polymerase," Science, vol. 239, no. 4839, pp. 487-491, 1988.

[17] B. Masala and L. Manca, "Detection of the common Hb F Sardinia $[(\mathrm{A}) \gamma(\mathrm{E} 19) \mathrm{Ile} \rightarrow \mathrm{Thr}]$ variant by isoelectric focusing in normal newborns and in adults affected by elevated fetal hemoglobin syndromes," Clinica Chimica Acta, vol. 198, no. 3 , pp. 195-202, 1991.

[18] G. D. Efremov, B. Markovska, N. Stojanovski, G. Petkov, N. Nikolov, and T. H. J. Huisman, "The use of globin chain electrophoresis in polyacrylamide gels for the quantitation of the ${ }^{G} \gamma$ to ${ }^{A} \gamma$ ratio in fetal hemoglobin," Hemoglobin, vol. 5, no. 7-8, pp. 637-651, 1981.

[19] L. Manca, M. Formato, P. Demuro, D. Gallisai, M. Orzalesi, and B. Masala, "The gamma globin chain heterogeneity of the Sardinian newborn baby," Hemoglobin, vol. 10, no. 5, pp. 519528, 1986.

[20] M. Braend, L. L. Nesse, and G. D. Efremov, "Expression and genetics of caprine haemoglobins," Animal Genetics, vol. 18, no. 3, pp. 223-231, 1987.

[21] B. Giardina and G. Amiconi, "Measurement of binding of gaseous and nongaseous ligands to hemoglobins by conventional spectrophotometric procedures," Methods in Enzymology, vol. 76, pp. 417-427, 1981.

[22] K. Imai, Allosteric Effects in Haemoglobin, Cambridge University Press, Cambridge, Mass, USA, 1982.

[23] G. Fermi, M. F. Perutz, B. Shaanan, and R. Fourme, "The crystal structure of human deoxyhaemoglobin at $1.74 \AA$ resolution," Journal of Molecular Biology, vol. 175, no. 2, pp. 159-174, 1984.

[24] M. Ceccarelli, R. Anedda, M. Casu, and P. Ruggerone, "CO escape from myoglobin with metadynamics simulations," Proteins, vol. 71, no. 3, pp. 1231-1236, 2008.

[25] M. A. Scorciapino, A. Robertazzi, M. Casu, P. Ruggerone, and M. Ceccarelli, "Breathing motions of a respiratory protein revealed by molecular dynamics simulations," Journal of the American Chemical Society, vol. 131, no. 33, pp. 11825-11832, 2009.

[26] M. A. Scorciapino, A. Robertazzi, M. Casu, P. Ruggerone, and M. Ceccarelli, "Heme proteins: the role of solvent in the dynamics of gates and portals," Journal of the American Chemical Society, vol. 132, no. 14, pp. 5156-5163, 2010.

[27] B. Masala and L. Manca, "Detection of globin chains by reversed-phase high-performance liquid chromatography," Methods in Enzymology, vol. 231, pp. 21-44, 1994.

[28] S. Takahashi, A. K. L. C. Lin, and C. Ho, "Proton nuclear magnetic resonance studies of hemoglobins M Boston ( $\alpha 58 \mathrm{E} 7$ $\mathrm{His} \rightarrow \mathrm{Tyr})$ and M Milwaukee $(\beta 67 \mathrm{E} 11 \mathrm{Val} \rightarrow \mathrm{Glu}):$ spectral assignments of hyperfine-shifted proton resonances and of proximal histidine (E7) $\mathrm{NH}$ resonances to the $\alpha$ and $\beta$ chains of normal human adult hemoglobin," Biochemistry, vol. 19, no. 23, pp. 5196-5202, 1980. 
[29] T. Y. Fang, V. Simplaceanu, C. H. Tsai, N. T. Ho, and C. Ho, "An additional $\mathrm{H}$-bond in the $\alpha \beta$ interface as the structural basis for the low oxygen affinity and high cooperativity of a novel recombinant hemoglobin $(\beta \mathrm{L} 105 \mathrm{~W})$," Biochemistry, vol. 39, no. 45, pp. 13708-13718, 2000.

[30] V. Simplaceanu, J. A. Lukin, T. Y. Fang, M. Zou, N. T. Ho, and C. Ho, "Chain-selective isotopic labeling for NMR studies of large multimeric proteins: application to hemoglobin," Biophysical Journal, vol. 79, no. 2, pp. 1146-1154, 2000.

[31] M. F. Perutz, "Stereochemistry of cooperative effects in haemoglobin," Nature, vol. 228, no. 5273, pp. 726-739, 1970.

[32] L. W. M. Fung and C. Ho, "A proton nuclear magnetic resonance study of the quaternary structure of human hemoglobins in water," Biochemistry, vol. 14, no. 11, pp. 25262535, 1975.

[33] K. Ishimori, K. Imai, G. Miyazaki et al., "Site-directed mutagenesis in hemoglobin: functional and structural role of inter- and intrasubunit hydrogen bonds as studied with $37 \beta$ and $145 \beta$ mutations," Biochemistry, vol. 31, no. 12, pp. 32563264, 1992.

[34] K. Imai, H. Morimoto, M. Kotani, S. Shibata, T. Miyaji, and K. Matsutomo, "Studies on the function of abnormal hemoglobins II. Oxygen equilibrium of abnormal hemoglobins: Shimonoseki, Ube II, Hikari, Gifu, and Agenogi," Biochimica et Biophysica Acta, vol. 200, no. 2, pp. 197-202, 1970.

[35] R. Mojzikova, P. Dolezel, J. Pavlicek, P. Mlejnek, D. Pospisilova, and V. Divoky, "Partial glutathione reductase deficiency as a cause of diverse clinical manifestations in a family with unstable hemoglobin (Hemoglobin Haná, $\beta 63$ (E7) His-Asn)," Blood Cells, Molecules, and Diseases, vol. 45, no. 3, pp. 219-222, 2010. 


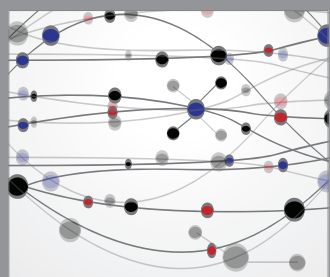

The Scientific World Journal
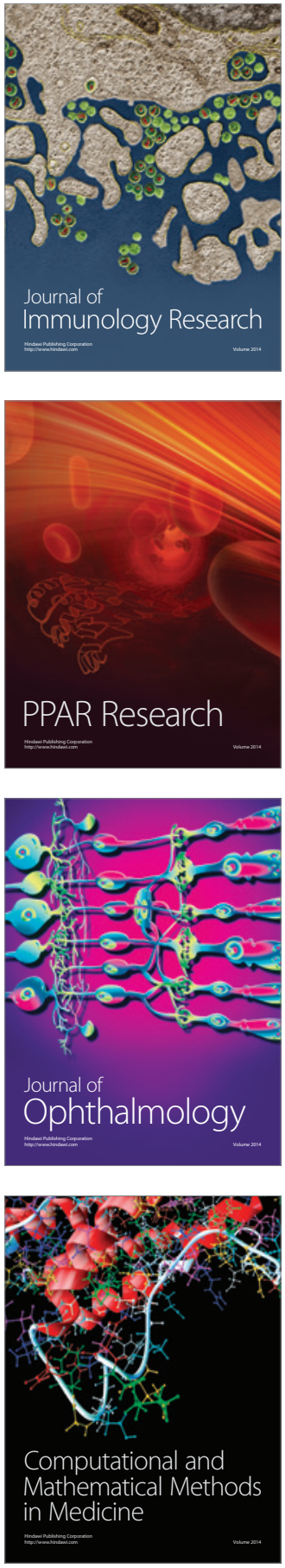

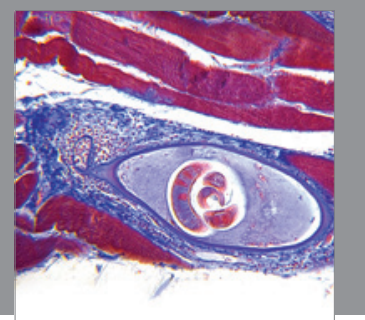

Gastroenterology

Research and Practice
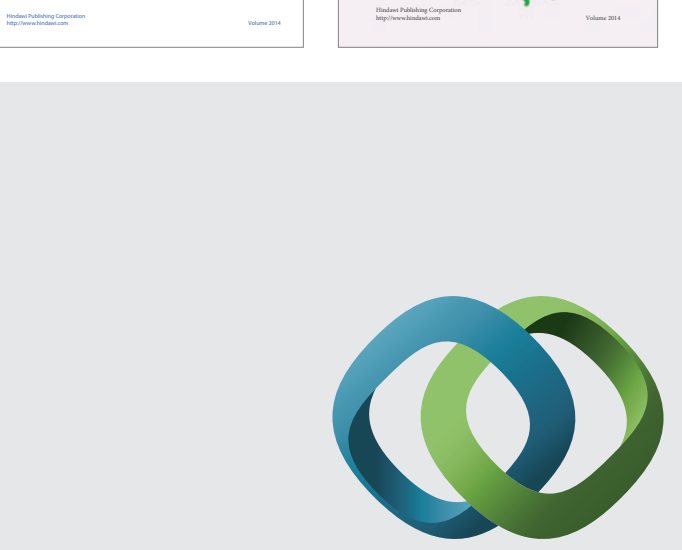

\section{Hindawi}

Submit your manuscripts at

http://www.hindawi.com
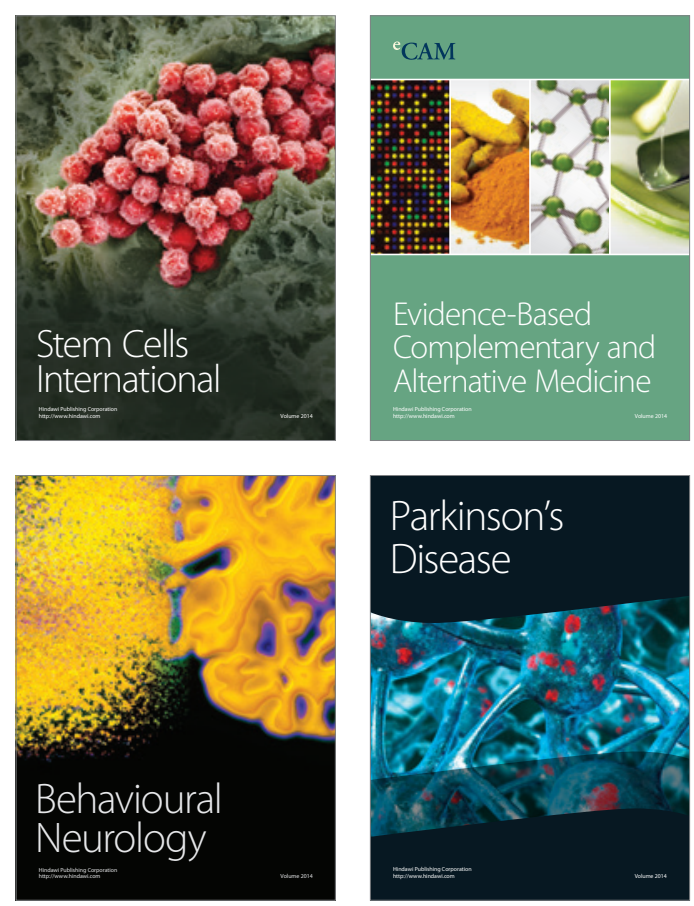

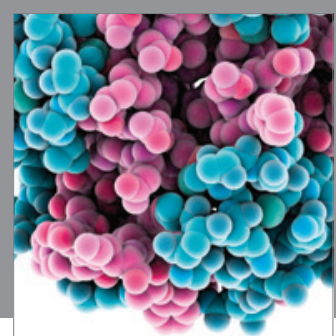

Journal of
Diabetes Research

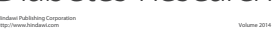

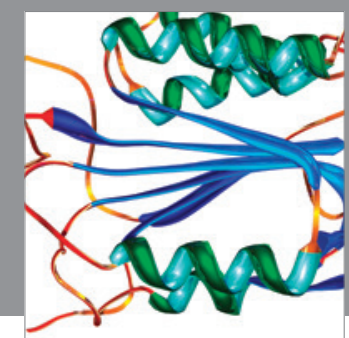

Disease Markers
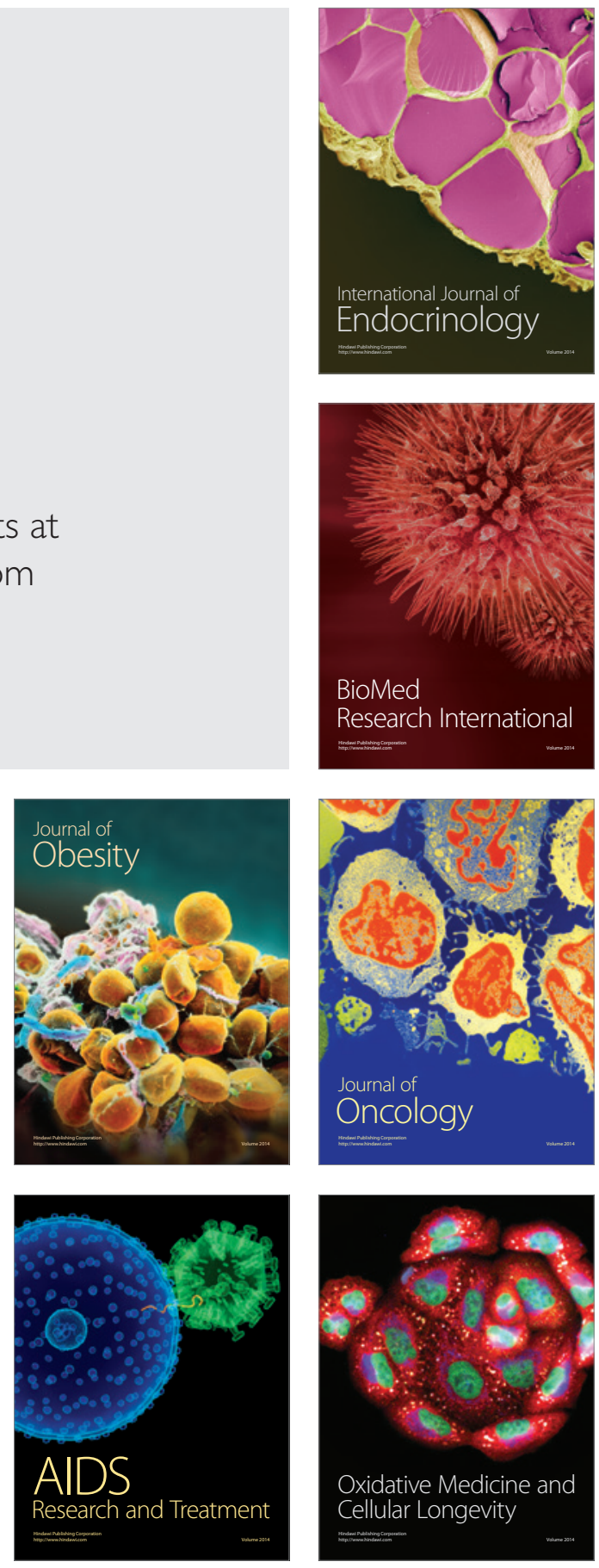